\title{
In vitro investigation of the effects of tryptophan fermentation products on immune response
}

\author{
L. Abecia, A. L. Mccartney and A. Klinder \\ Food Microbial Science Unit, School of Chemistry, Food Biosciences and Pharmacy, University of Reading, RG6 6AP, UK
}

Autism spectrum disorders (ASD) are a group of relatively common neurodevelopmental disorders affecting between 2 and 6 children per 1000. The aetiology of autism is unknown with both environmental and genetic factors considered to play key roles. Different research groups have described various immune abnormalities, including increased levels of inflammatory mediators and the presence of autoimmune phenomena. The intestinal microbiota has marked influences on the intestinal and peripheral host's immunity and differences between ASD and healthy children's microbiota have been reported. Tryptophan (TRP) is an essential amino acid that is preferentially acquired from the diet and is the precursor of a wide range of biologically active substances such as serotonin, melatonin and kynurenine, the role of this metabolic pathway in autism deserves more detailed analysis.

The aim of this study was to investigate the effect of the fermentation products of TRP, obtained from in vitro systems for ASD volunteers $(n=3)$ and age-matched typically developing controls (TDC, $n=3$ ) microbiota, on the immune response of an in vitro model of colon.

$\mathrm{pH}$-controlled faecal batch single-stage continuous culture fermentations were used to obtain in vitro fermentation products of TRP. Fermentation basal medium was supplemented with $1 \%(\mathrm{w} / \mathrm{v})$ L-tryptophan and $\mathrm{pH}$ adjusted to 6.8. Each vessel was inoculated with $10 \mathrm{ml}$ of fresh $10 \%$ (w/v) faecal slurry from 3 ASD children or 3 TDC (from 5 to 13 years old). After $24 \mathrm{~h}$ of fermentation, continuous feeding was applied to the vessels at two different flow rates (fast or slow) to simulate 20 and $40 \mathrm{~h}$ of retention time, respectively. Samples were taken after $312 \mathrm{~h}$ of running experiment and centrifuged to remove the bacteria and then filtered-sterilized. HT-29 cells were incubated for $24 \mathrm{~h}$ with Dulbecco's modified Eagle medium (DMEM) media plus $10 \%$ of each supernatant obtained from the previous fermentations. Total RNA was isolated from cells by using the monophasic phenol/guanidine isothiocyanate solution extraction method (TRIzol ${ }^{\mathrm{TM}}$ reagent), reversely transcribed (SuperScript ${ }^{\mathrm{TM}}$ III First-Strand Synthesis SuperMix kit, Invitrogen) and relative expression levels of mRNA determined by real time RT-PCR assays using PRISM 7300 Sequence Detection system. Relative expression levels were normalised with respect to expression levels of untreated (control) cells (fold change $=1$ ) using the Pfaffl method. The housekeeping gene used was GAPDH. All samples were compared for each gene using GLM procedure of SAS version 9 with a split plot design (Table 1).

Table 1. Relative fold change of IgA1 and NF- $\kappa \beta$ gene expression in HT-29 cells after $24 \mathrm{~h}$ incubation with TRP metabolites

\begin{tabular}{|c|c|c|c|c|c|c|c|c|}
\hline & \multicolumn{2}{|c|}{ ASD } & \multicolumn{2}{|c|}{ TDC } & \multirow[b]{2}{*}{$\mathrm{SE}^{1}$} & \multirow[b]{2}{*}{$\mathrm{SE}^{2}$} & \multicolumn{2}{|c|}{ Significance } \\
\hline & Fast & Slow & Fast & Slow & & & Volunteer & Flow rate \\
\hline$\overline{\operatorname{Ig} A 1}$ & $0.10^{\mathrm{a}}$ & $0.83^{\mathrm{a}}$ & $-0.67^{b}$ & $-0.40^{\mathrm{b}}$ & 0.26 & 0.85 & * & NS \\
\hline $\mathrm{NF}-\kappa \beta$ & -1.57 & -0.44 & 0.57 & -0.33 & 1.24 & 0.28 & NS & NS \\
\hline
\end{tabular}

$\mathrm{SE}^{1}$, volunteer (main plot) error; $\mathrm{SE}^{2}$, flow rate (split-plot) error. A $P$ value of $<0.05$ is shown as *.

$\mathrm{NF}-\kappa \beta$ is a transcriptional factor that intervenes in the synthesis of the proinflammatory cytokines by cells in the innate immune system and intestinal enterocytes. On the other hand, IgA belongs to the acquired immunity. Differences in the microbial composition of different systems may catabolise TRP using different pathways. The response of NF- $\kappa \beta$ and IgA1 to TRP metabolites was considered weak. However, there was a significant difference in IgA expression which was up-regulated in the systems inoculated with ASD faecal microbiota. Flow rate did not affect gene expression. IgA response was related to Clostridium histolyticum. Interestingly, this group of gut bacteria was significantly higher in ASD children. Further investigation is necessary to elucidate the relationship between immunological findings, microbiota and behaviour in autism. 